\title{
SOME UNBOUNDED FUNCTIONS OF REGULAR GROWTH
}

\author{
H. ARTHUR DEKLEINE AND VLADIMIR DROBOT
}

\begin{abstract}
The concept of regular growth for unbounded nondecreasing functions has its origin in the study of the asymptotic behavior of solutions for the second order equation $u^{\prime \prime}+a(t) u=0$. In this paper we give sufficient conditions for a continuous, differentiable function $a(t)$ to possess the property that its logarithm increases regularly. We also show that the logarithm of a continuous unbounded concave or convex function increases regularly.
\end{abstract}

The asymptotic behavior of solutions of the differential equation

$$
u^{\prime \prime}+a(t) u=0,
$$

where $a(t)$ is a positive, nondecreasing, continuous function such that $a(t) \rightarrow+\infty$ as $t \rightarrow+\infty$, has been extensively studied. It is well known that the stated conditions on $a(t)$ are not sufficient to conclude that all solutions of (1) satisfy

$$
u(t) \rightarrow 0 \text { as } t \rightarrow+\infty
$$

see for example D. Willett [10] and H. A. DeKleine [3]. Sufficient conditions assuring that all solutions of (1) satisfy (2) have been obtained by G. Armellini and independently by L. Tonelli and G. Sansone (cf. L. Cesari [2, p. 85] and G. Sansone [9, p. 61]).

Let $f(t)$ be a positive, continuous, nondecreasing function whose domain is some half line $t_{0} \leqq t<\infty$ with $f(t) \rightarrow+\infty$. We say that $f(t)$ grows intermittently or quasi-jumping if for every $\varepsilon>0$ there is an unbounded sequence $t_{0} \leqq \alpha_{1}<\beta_{1}<\alpha_{2}<\beta_{2}<\cdots$,

and

$$
\underset{n \rightarrow \infty}{\lim \sup } \sum_{k=1}^{n}\left(\beta_{k}-\alpha_{k}\right) / \beta_{n} \leqq \varepsilon
$$

$$
\sum_{k=1}^{\infty}\left[f\left(\alpha_{k+1}\right)-f\left(\beta_{k}\right)\right]<\infty
$$

If this does not occur, we say that $f(t)$ grows regularly.

Presented to the Society, January 17, 1972; received by the editors August 23, 1971 and, in revised form, November 29, 1971.

AMS 1970 subject classifications. Primary 34C10; Secondary 26A48.

Key nords and phrases. Asymptotic behavior, concave, convex, regular growth.

(c) American Mathematical Society 1972 
Theorem 1 (Armellini, Tonelli, Sansone). If $a(t)$ is a positive, unbounded, nondecreasing, continuous function such that $\log a(t)$ grows regularly, then every solution of (1) satisfies (2).

In the original statement of this result, $a(t)$ was required to be continuously differentiable; however, it is sufficient to require only that $a(t)$ be continuous. P. Hartman [4] gave a simple proof of this more general result.

More recently, A. Meir, D. Willett, and J. S. W. Wong [6] obtained the following:

Theorem 2 [6, COROLlary 1]. Let a $(t)$ be a positive, unbounded, nondecreasing function in $C^{1}[0, \infty)$. If there exists a positive, nondecreasing function $p(t) \in C^{1}[0, \infty)$ such that

$$
\int_{0}^{\infty}[p(t)]^{-1} d t=+\infty
$$

and

$$
\liminf _{t \rightarrow+\infty} a^{\prime}(t) p(t) / a(t)>0
$$

then (2) holds for all solutions of (1).

In fact, A. Meir, D. Willett and J. S. W. Wong established a more general result than Theorem 2 in which the condition that $p(t)$ is increasing is replaced by the less demanding requirement that

$$
\liminf _{t \rightarrow \infty} p^{\prime}(t) / p(t) a^{1 / 2}(t) \geqq 0 .
$$

They show that Theorem 2, as stated above, is a corollary of a well known result by G. Sansone [9, p. 65].

The aim of this note is to show that any function $a(t)$ satisfying the conditions of Theorem 2 has the property that its logarithm is of regular growth, hence Theorem 2 is a corollary of Theorem 1 .

THEOREM 3. Let $a(t)$ be a positive, unbounded, nondecreasing function in $C^{1}[0, \infty)$. If there exists a positive, nondecreasing, continuous function $p(t)$ such that

and

$$
\int_{0}^{\infty}[p(t)]^{-1} d t=\infty
$$

$$
\liminf _{t \rightarrow \infty} a^{\prime}(t) p(t) / a(t)=2 \delta>0,
$$

then $\log a(t)$ increases regularly. 
Proof of Theorem 3. Let $\varepsilon$ be any number strictly smaller than $\frac{1}{2}$. Let $\alpha_{1}<\beta_{1}<\alpha_{2}<\beta_{2}<\cdots$ be any unbounded sequence such that

$$
\limsup _{n \rightarrow \infty} \sum_{k=1}^{n}\left(\beta_{k}-\alpha_{k}\right) / \beta_{n} \leqq \varepsilon .
$$

There is no loss in generality in assuming that $a^{\prime}(t) / a(t) \geqq \delta / p(t)$ for $t \geqq \alpha_{1}$. We need only show that equation (4) is satisfied with $f(t)=\log a(t)$.

First, however, we see that for some $L \geqq 2$

$$
\sum_{k=1}^{n-1}\left[\alpha_{k+1}-\beta_{k}\right]>\sum_{k=1}^{n}\left[\beta_{k}-\alpha_{k}\right]
$$

for $n \geqq L$. If this were not the situation, it would necessarily follow from (3) that $\lim _{n \rightarrow \infty} \sum_{k=1}^{n}\left[\beta_{k}-\alpha_{k}\right]=+\infty$ and that

$$
\underset{n \rightarrow \infty}{\lim \sup } \sum_{k=1}^{n}\left(\beta_{k}-\alpha_{k}\right) / \beta_{n} \geqq \frac{1}{2} .
$$

If $\sum_{k=1}^{\infty}\left[\beta_{k}-\alpha_{k}\right]=\infty$, we define two functions $p_{1}(t)$ and $p_{2}(t)$, having $[0, \infty)$ as their domain, by

$$
p_{1}(t)=p\left(t+\sum_{k=1}^{N}\left[\alpha_{k+1}-\beta_{k}\right]+\alpha_{1}\right),
$$

where $N=\max \left\{n: 0 \leqq n\right.$ and $\left.\sum_{k=1}^{n}\left[\beta_{k}-\alpha_{k}\right] \leqq t\right\}$, and

$$
p_{2}(t)=p\left(t+\sum_{k=1}^{M}\left[\beta_{k}-\alpha_{k}\right]+\alpha_{1}\right)
$$

where $M=\max \left\{n: 1 \leqq n\right.$ and $\left.\sum_{k=1}^{n-1}\left[\alpha_{k+1}-\beta_{k}\right] \leqq t\right\}$. By taking note of the fact that

and that

$$
t+\sum_{k=1}^{N}\left[\alpha_{k+1}-\beta_{k}\right]+\alpha_{1}=t-\sum_{k=1}^{N}\left[\beta_{k}-\alpha_{k}\right]+\alpha_{N+1}
$$

$$
t+\sum_{k=1}^{M}\left[\beta_{k}-\alpha_{k}\right]+\alpha_{1}=t-\sum_{k=1}^{M-1}\left[\alpha_{k+1}-\beta_{k}\right]+\beta_{M}
$$

we have the following inequalities:

$$
\begin{gathered}
\alpha_{N+1} \leqq t+\sum_{k=1}^{N}\left[\alpha_{k+1}-\beta_{k}\right]+\alpha_{1}<\beta_{N+1}, \\
\beta_{M} \leqq t+\sum_{k=1}^{M}\left[\beta_{k}-\alpha_{k}\right]+\alpha_{1}<\alpha_{M+1}
\end{gathered}
$$


Recall that $p(t)$ is nondecreasing. Hence, for $t \geqq T=\sum_{k=1}^{L-1}\left[\alpha_{k+1}-\beta_{k}\right]$,

$$
\left[p_{1}(t)\right]^{-1} \leqq\left[p\left(\alpha_{N+1}\right)\right]^{-1} \leqq\left[p\left(\alpha_{M+1}\right)\right]^{-1} \leqq\left[p_{2}(t)\right]^{-1} .
$$

We are now in a position to obtain the desired result. If $\sum_{k=1}^{\infty}\left[\beta_{k}-\alpha_{k}\right]<$ $\infty$, we have

$$
\sum_{k=1}^{\infty} \int_{\beta_{k}}^{\alpha_{k+1}}[p(\tau)]^{-1} d \tau=\int_{\alpha_{1}}^{\infty}[p(\tau)]^{-1} d \tau-\sum_{k=1}^{\infty} \int_{\alpha_{k}}^{\beta_{k}}[p(\tau)]^{-1} d \tau=\infty .
$$

If $\sum_{k=1}^{\infty}\left[\beta_{k}-\alpha_{k}\right]=\infty$, then

$$
\begin{aligned}
\sum_{k=1}^{\infty} \int_{\beta_{k}}^{\alpha_{k+1}}[p(\tau)]^{-1} d \tau & =2 \int_{0}^{\infty} p_{2}(\tau) d \tau \\
& \geqq C_{0}+\int_{0}^{\infty}\left[p_{1}(\tau)\right]^{-1} d \tau+\int_{0}^{\infty}\left[p_{2}(\tau)\right]^{-1} d \tau \\
& =C_{0}+\int_{0}^{\infty}[p(\tau)] d \tau=\infty
\end{aligned}
$$

where $C_{0}=\int_{0}^{T}\left[p_{2}(\tau)\right]^{-1} d \tau-\int_{0}^{T}\left[p_{1}(\tau)\right]^{-1} d \tau$. Therefore

$$
\begin{aligned}
\sum_{k=1}^{\infty} \log a\left(\alpha_{k+1}\right)-\log a\left(\beta_{k}\right) & =\sum_{k=1}^{\infty} \int_{\beta_{k}}^{\alpha_{k+1}} a^{\prime}(\tau) / a(\tau) d \tau \\
& \geqq \delta \sum_{k=1}^{\infty} \int_{\beta_{k}}^{\alpha_{k+1}}[p(\tau)]^{-1} d \tau=\infty,
\end{aligned}
$$

as is required for the proof of the theorem.

THEOREM 4. If $a(t)$ is a positive, unbounded, nondecreasing, continuous function and either $a(t)$ is concave or convex, then $\log a(t)$ increases regularly.

COROllaRY. (SEE [6].) If a(t) satisfies the hypothesis of Theorem 4, then every solution of (1) satisfies (2).

Proof of THEOREM 4. Let $\varepsilon$ be any number strictly smaller than $\frac{1}{2}$. Let $\alpha_{1}<\beta_{1}<\alpha_{2}<\beta_{2}<\cdots$ be an unbounded sequence such that $\lim \sup _{n \rightarrow \infty} \sum_{k=1}^{n}\left(\beta_{k}-\alpha_{k}\right) / \beta_{n} \leqq \varepsilon$ Let $L$ and $T$ be defined as in the proof of Theorem 3 . In either case we define a piecewise linear function $\lambda$ by

$$
\begin{array}{ll}
\lambda(t)=\frac{t-\alpha_{k}}{\beta_{k}-\alpha_{k}} a\left(\beta_{k}\right)+\frac{\beta_{k}-t}{\beta_{k}-\alpha_{k}} a\left(\alpha_{k}\right), & \alpha_{k} \leqq t \leqq \beta_{k}, \\
\lambda(t)=\frac{t-\beta_{k}}{\alpha_{k+1}-\beta_{k}} a\left(\alpha_{k+1}\right)+\frac{\alpha_{k+1}-t}{\alpha_{k+1}-\beta_{k}} a\left(\beta_{k}\right), & \beta_{k} \leqq t \leqq \alpha_{k+1} .
\end{array}
$$


Let us first consider the case when $a(t)$ is concave. In this case $\lambda(t)$ is also concave and $\lambda^{\prime}(t) / \lambda(t)$ is decreasing excluding, of course, the isolated points where $\lambda^{\prime}$ is undefined. Using an argument analogous to the one used in the proof of Theorem 3,

Therefore,

$$
\sum_{k=1}^{\infty} \int_{\beta_{k}}^{\alpha_{k+1}} \lambda^{\prime}(\tau) / \lambda(\tau) d \tau=\infty
$$

$$
\begin{aligned}
\sum_{k=1}^{\infty}\left\{\log a\left(\alpha_{k+1}\right)-\log a\left(\beta_{k}\right)\right\} & =\sum_{k=1}^{\infty}\left\{\log \lambda\left(\alpha_{k+1}\right)-\log \lambda\left(\beta_{k}\right)\right\} \\
& =\sum_{k=1}^{\infty} \int_{\beta_{k}}^{\alpha_{k+1}} \lambda^{\prime}(\tau) / \lambda(\tau) d \tau=\infty
\end{aligned}
$$

Now consider the case when $a(t)$ is convex. In this case $\lambda(t)$ is also convex and unbounded. Since, for $t$ not equal to any $\alpha_{k}$ or $\beta_{k}$,

$$
\lambda(t)-\lambda\left(\alpha_{1}\right)=\int_{\alpha_{1}}^{t} \tau \lambda^{\prime}(\tau) / \tau d \tau \leqq t \lambda^{\prime}(t) \log \left(t / \alpha_{1}\right),
$$

we have that

$$
\lim _{t \rightarrow \infty} t \log \left(t / \alpha_{1}\right) \lambda^{\prime}(t) / \lambda(t)=1
$$

Let $\Gamma$ be some positive integer such that $\lambda^{\prime}(t) / \lambda(t) \geqq \frac{1}{2}\left[t \log \left(t / \alpha_{1}\right)\right]^{-1}$ for $t \geqq \beta_{\Gamma}$. Again, using an argument analogous to the one used in the proof of Theorem 3,

$$
\sum_{k=1}^{\infty} \int_{\beta_{k}}^{\alpha_{k+1}}\left[\tau \log \left(\tau / \alpha_{1}\right)\right]^{-1} d \tau=\infty
$$

Therefore,

$$
\begin{aligned}
\sum_{k=1}^{\infty}\left\{\log a\left(\alpha_{k+1}\right)-\log a\left(\beta_{k}\right)\right\} & =\sum_{k=1}^{\infty}\left\{\log \lambda\left(\alpha_{k+1}\right)-\log \lambda\left(\beta_{k}\right)\right\} \\
& =\sum_{k=1}^{\infty} \int_{\beta_{k}}^{\alpha_{k+1}} \lambda^{\prime}(\tau) / \lambda(\tau) d \tau \\
& \geqq C_{1}+\frac{1}{2} \sum_{k=1}^{\infty} \int_{\beta_{k}}^{x_{k+1}}\left[\tau \log \left(\tau / \alpha_{1}\right)\right]^{-1} d \tau=\infty
\end{aligned}
$$

where

$$
C_{1}=\sum_{k=1}^{\Gamma}\left\{\int_{\beta_{k}}^{\alpha_{k+1}} \lambda^{\prime}(\tau) / \lambda(\tau) d \tau-\int_{\beta_{k}}^{\alpha_{k+1}}\left[\tau \log \left(\tau / \alpha_{1}\right)\right]^{-1} d \tau\right\} .
$$

Hence our desired result. 


\section{REFERENCES}

1. I. Bihari, Extension of a theorem of Armellini-Tonelli-Sansone to the nonlinear equation $u^{\prime \prime}+a(t) f(u)=0$, Magyar Tud. Akad. Mat. Kutató Int. Közl. 7 (1962), 63-68. MR 26 \#6498.

2. L. Cesari, Asymptotic behavior and stability problems in ordinary differential equations, Ergebnisse der Mathematik und ihrer Grenzgebiete, N. F., Heft 16, SpringerVerlag, Berlin, 1959. MR 22 \#9673.

3. H. A. DeKleine, A counterexample to a conjecture in second-order linear equations, Michigan Math. J. 17 (1970), 29-32. MR 41 \#5690.

4. P. Hartman, On oscillators with large frequencies, Boll. Un. Mat. Ital. (3) 14 (1959), 62-65. MR 21 \#3610.

5. - The existence of large or small solutions of linear differential equations, Duke Math. J. 28 (1961), 421-429. MR 24 \#A293.

6. A. Meir, D. Willett and J. S. W. Wong, On the asymptotic behavior of the solutions of $x^{\prime \prime}+a(t) x=0$, Michigan Math. J. 14 (1967), 47-52. MR 35 \#463.

7. E. J. McShane, On the solutions of the differential equation $y^{\prime \prime}+p^{2} y=0$, Proc. Amer. Math. Soc. 17 (1966), 55-61. MR 32 \#5991.

8. Z. Opial, Sur l'équation différentielle $u^{\prime \prime}+a(t) u=0$, Ann. Polon. Math. 5 (1958), 77-93. MR 20 \#4047.

9. G. Sansone, Equazioni differenziali nel campo reale, Vol. 2, 2nd ed., Nicola Zanichelli, Bologna, 1949. MR 11, 32.

10. D. Willett, On an example in second order linear ordinary differential equations, Proc. Amer. Math. Soc. 17 (1966), 1263-1266. MR 34 \#1612.

Department of Mathematics, State University of New York at Buffalo, AMHERST, NeW York 14226 